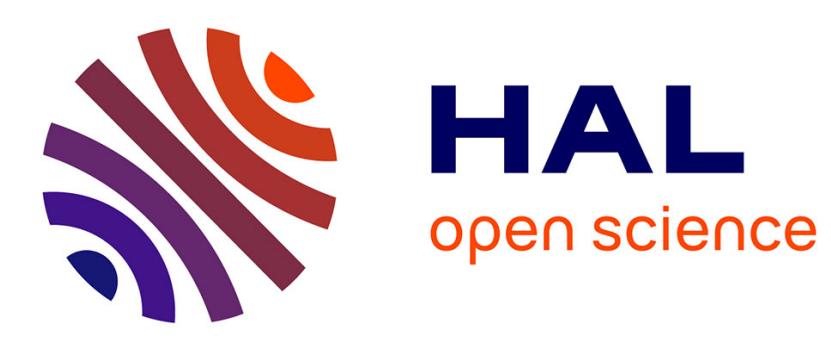

\title{
Partage de la mer et nouveaux conflits géopolitiques en Baltique
}

\author{
Nicolas Escach Escach
}

\section{To cite this version:}

Nicolas Escach Escach. Partage de la mer et nouveaux conflits géopolitiques en Baltique. Hérodote - Revue de géographie et de géopolitique, 2016, Mers et Océans, 163, pp.175-191. 10.3917/her.163.0175. halshs-01470238v2

\section{HAL Id: halshs-01470238 \\ https://shs.hal.science/halshs-01470238v2}

Submitted on 3 May 2017

HAL is a multi-disciplinary open access archive for the deposit and dissemination of scientific research documents, whether they are published or not. The documents may come from teaching and research institutions in France or abroad, or from public or private research centers.
L'archive ouverte pluridisciplinaire HAL, est destinée au dépôt et à la diffusion de documents scientifiques de niveau recherche, publiés ou non, émanant des établissements d'enseignement et de recherche français ou étrangers, des laboratoires publics ou privés. 
Nicolas Escach

Docteur et agrégé en géographie

ATER à l'IEP de Rennes

Campus Bretagne (Rennes) et Normandie (Caen)

Membre de l'UMR-5600 EVS

\title{
Partage de la mer et nouveaux conflits géopolitiques en Baltique \\ Sharing the sea and new geopolitical conflicts in the Baltic region
}

\section{Résumé}

Les réseaux de coopération qui se sont multipliés en mer Baltique depuis la chute du Rideau de fer conduiraient presque à la considérer comme un lac dépourvu de la moindre fracture. D'importants conflits s'y sont pourtant succédé révélant sa situation stratégique à l'échelle européenne. La guerre froide l'a même érigé en no man's land militarisé, confortant son rôle de glacis entre les deux blocs. Si la présence de l'OTAN dans la zone semble désormais écarter la menace d'un futur conflit armé, des tensions de faible intensité, discontinues dans l'espace et le temps, s'accumulent afin de redessiner les lignes de l'entre-deux géopolitique dans les failles d'une régionalisation incomplète. La mer représente non seulement un théâtre mais aussi un enjeu croissant pour ces nouvelles rivalités à mesure que s'accroît l'appropriation du territoire liquide. Les confrontations maritimes traduisent en Baltique un regain de conflictualité aux antipodes de l'image d'une gouvernance apaisée et exemplaire.

\begin{abstract}
The network of cooperation which has multiplied in the Baltic Sea since the fall of the Iron Curtain could almost be compared to a frozen lake without the smallest crack. The important conflicts there have nonetheless successfully revealed its strategic position on the European scale. The Cold War effectively built it into a militarized "no man's land," solidifying its role as a buffer between the two blocks. Even though NATO's presence in the zone appears to diffuse the threat of a future armed conflict, the slight underlying tensions are accumulating, redrawing the lines of the geopolitical divide, further pushing the regions apart. The Sea not only represents a stage but also ever higher stakes for the new rivalries, impacted by the increased appropriation of liquid territory. The maritime confrontations translate in the Baltic region into renewed conflict which is the antithesis of the image of a peaceful and exemplary coalition.
\end{abstract}

L'auteur tient à remercier Madison Ryckman pour l'aide apportée à la traduction.

\section{Introduction}

L'espace baltique a souvent été cité en exemple par les autorités européennes depuis la fin des années 1990 comme un laboratoire propice à l'expérimentation de nouvelles pratiques communautaires [Commission européenne, 1994 ; Commission européenne, 1999]. L'existence de liens transnationaux anciens, réactivés après la chute du Rideau de fer, a même conduit certains chercheurs à évoquer un modèle de gouvernance transnationale [Christiansen, 1997]. La singularité géographique de la Baltique, neuf États riverains se faisant face autour d'une mer 
quasiment fermée (superficie réduite, verrou des détroits danois, faible renouvellement des eaux, englacement), inviterait tantôt à la rapprocher d'une méditerranée [Brunet, 1995 ; Dollfus, 1995], tantôt d'un lac [Joenniemi, 2009].

Longtemps glacis sur la frontière ouest/est, la mer Baltique est devenue, au cours des décennies 1980-1990, un support et un moteur pour les réseaux de coopération entre acteurs de tous niveaux (municipalités et ports, régions, États). Signée en 1974 et révisée en 1992, la Convention pour la protection du milieu marin de la mer Baltique, aussi appelée Convention d'Helsinki, est le premier texte-cadre d'envergure rédigé par les États riverains ${ }^{1}$ alors engagés dans la guerre froide [Rydén, 2002]. L'article 1 précise que l'aire à protéger comprend la Baltique propre à laquelle s'ajoutent les golfes de Finlande et de Botnie [HELCOM, 1974]. La réunification imparfaite des deux rives doublée d'une intégration partielle au sein de l'Union européenne ${ }^{2}$ ont ensuite généré une multitude de structures plus ou moins formelles sans qu'une institution dominante n'impose sa vision du développement régional [Ostergren, Le Bossé, 2005]. L'Union des villes de la Baltique (1991), le Conseil des États de la mer Baltique (1992), Vision et stratégies autour de la mer Baltique (1992) ou encore Baltic 21 (1996) en sont quelques exemples. À mesure que le champ lexical s'est «démilitarisé », un nouveau vocabulaire («voisins », «riverains ») a accompagné un discours performatif sur la Baltique comme espace de coopération, l'imaginaire de la rive succédant à celui du rivage ${ }^{3}$. Le besoin de compter dans une Union européenne en construction, des recherches opportunistes d'investissements ou la volonté d'accélérer l'harmonisation des structures économiques et juridiques, le tout teinté d'une coloration historique et culturelle, ont un temps masqué les divisions et les rancœurs. Olav Knudsen rappelle pourtant que « ce sont les conflits, plutôt que la coopération, qui ont rapproché les puissances qui entourent la mer Baltique » [Knudsen, 1999 : 9].

Trop souvent étudiée comme le théâtre secondaire voire collatéral de conflits qui la dépassent - en témoigne l'intérêt des médias pour les conséquences de la crise ukrainienne sur les relations russo-baltes - la mer Baltique n'est pas exempte de tensions géopolitiques à toutes les échelles. Les frontières étatiques s'y enchevêtrent sur à peine $385000 \mathrm{~km}^{2}$ traversés d'enclaves terrestres et maritimes. Les zones sous juridiction se superposent. Les ressources maritimes font l'objet d'une appropriation croissante sous le bleu apparemment lisse des cartes [Escach, 2014]. Ces dynamiques nous amènent à considérer la mer Baltique non seulement comme cadre mais aussi comme objet même des antagonismes interétatiques. Elles révèlent l'accumulation de tensions actives de faible intensité et leurs conséquences sur la recomposition en cours de l'ensemble de la zone.

\section{La Baltique : mer de guerre ou mer de paix?}

\section{Des conflits armés en héritage}

La mer Baltique a constitué une zone de front militaire pendant la plupart des conflits du XX siècle (Première Guerre mondiale, Seconde Guerre mondiale, guerre froide) comme en témoignent les 40000 tonnes d'armes chimiques encore enfouies au sud-est de Gotland, à l'est de l'île de Bornholm ${ }^{4}$ et dans le Skagerrak ou l'existence de nombreuses bases militaires et

\footnotetext{
${ }^{1}$ URSS, Pologne, RDA, Danemark, Suède, Finlande.

${ }^{2}$ Intégration du Danemark (1973), de la Suède et de la Finlande (1995), de la Pologne et des États baltes (2004).

${ }^{3}$ Les rives entourent un système introverti, clos et interconnecté. Le rivage, cher à Alain Corbin, désignerait plutôt l'immensité progressivement apprivoisée.

${ }^{4}$ Le bassin de Bornholm (105 mètres de profondeur) et la fosse de Gotland (459 mètres de profondeur) ont été des lieux privilégiés pour l'enfouissement des munitions chimiques comme du gaz moutarde après la décision prise
} 
villes fermées dont certaines sont aujourd'hui à l'abandon (voir figure $\mathrm{n}^{\circ} 1$ ). La rive orientale présente un «paysage du conflit » encore visible (friches militaires, glacis littoral, miradors et bunkers) dont l'île de Hiiumaa en Estonie et le port de Karosta en Lettonie nous donnent une idée plus précise.

\section{Figure $n^{\circ} 1$ : Enjeux géopolitiques et conflits en mer Baltique}

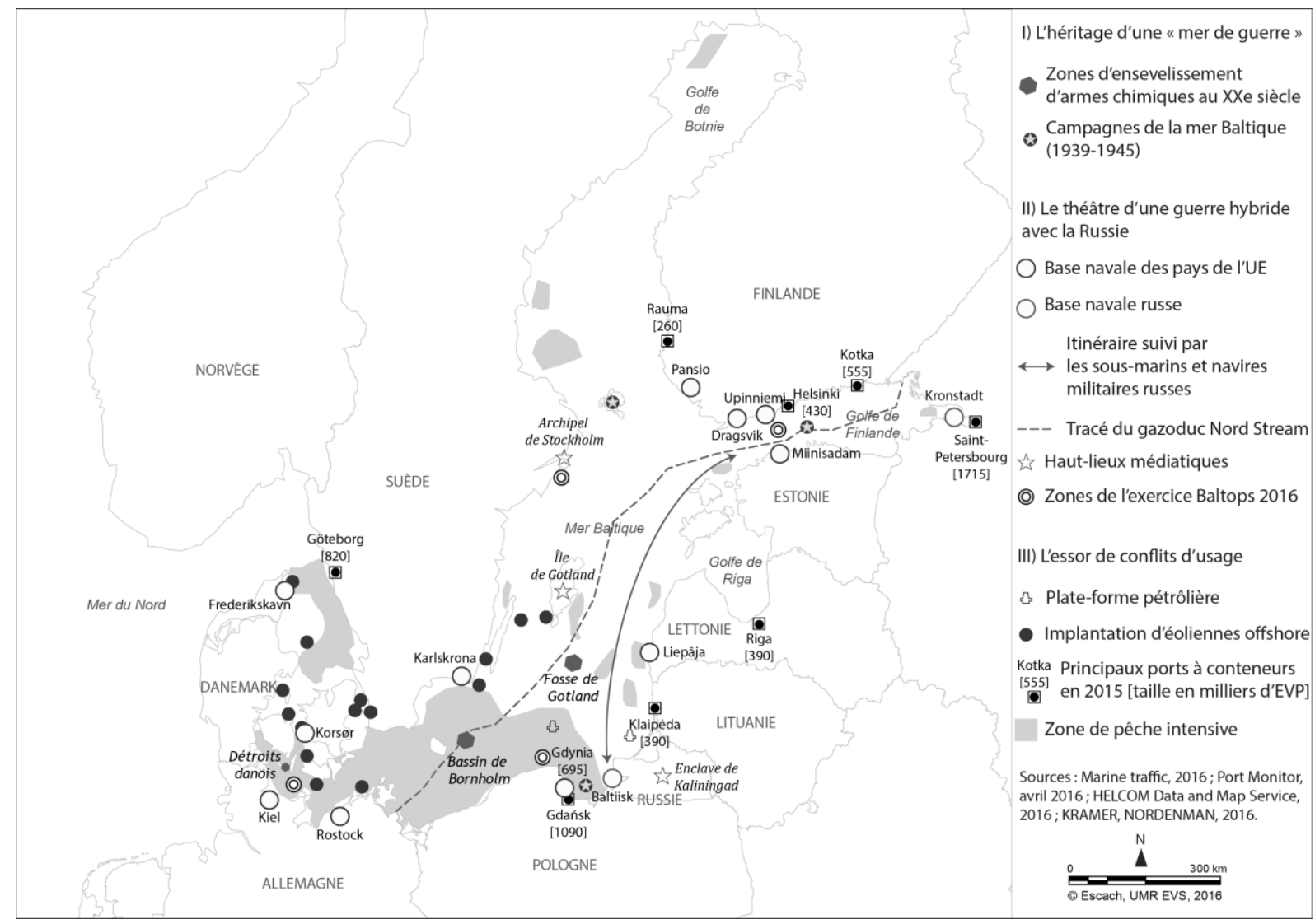

Hiiumaa, avec une superficie de $1000 \mathrm{~km}^{2}$, est la deuxième plus grande île d'Estonie après Saaremaa. L'île occupe une position stratégique qui a conduit à sa militarisation constante au cours du $\mathrm{XX}^{\mathrm{e}}$ siècle et donc à sa fermeture envers le reste de l'Europe (figure $\mathrm{n}^{\circ} 2$ ). Au cours de la Première Guerre mondiale, un vaste complexe de fortifications, la «forteresse navale de Pierre le Grand », est construit autour de Tallinn afin de protéger l'entrée du golfe de Finlande. Quatre lignes de défenses sont alors établies depuis Saint-Pétersbourg jusqu'à l'entrée du golfe, la dernière étant située entre Hiiumaa et la péninsule de Hanko en Finlande. L'île accueille ensuite des bâtiments militaires et de protection pendant la Seconde Guerre mondiale avant de devenir un élément clé du système de ceinture verte estonienne à l'époque soviétique. L'édification de nouvelles barrières de protection qui débute dès 1939 se prolonge donc jusque dans les années 1980. Le 26 octobre 1946, le conseil des ministres de la République socialiste soviétique d'Estonie vote une réglementation $\left(\mathrm{n}^{\circ} 058\right)$ établissant une ceinture littorale frontalière à accès restreint (restricted boarder coastal belt). Le 14 février 1955, celle-ci est étendue à toutes les îles estoniennes, à 24 conseils de village dans sept comtés à l'intérieur du pays et à la ville de Paldiski. Ces deux textes sont à l'origine d'une division du territoire estonien par zone (I, II, III, IV) en fonction du niveau de restriction. Hiiumaa, située en zone II, est accessible uniquement avec une permission émanant de la police militaire. L'île accueille

lors de la Conférence de Potsdam de détruire 267500 tonnes de bombes, missiles et mines contenant des armes chimiques. 
plusieurs bases militaires soviétiques notamment une base anti-aérienne sur la pointe de Tahkuna sur la rive nord. Aujourd'hui la pointe abrite le musée militaire de Hiiumaa et concentre la plupart des anciennes bases militaires et des bunkers de l'île, si l'on excepte les casernes et les tours de surveillance de la pointe occidentale de Ristna près du port de Kalana (péninsule de Kõpu).

\section{Figure $\mathrm{n}^{\circ} 2$ : un entre-deux géopolitique à l'entrée du golfe de Finlande}

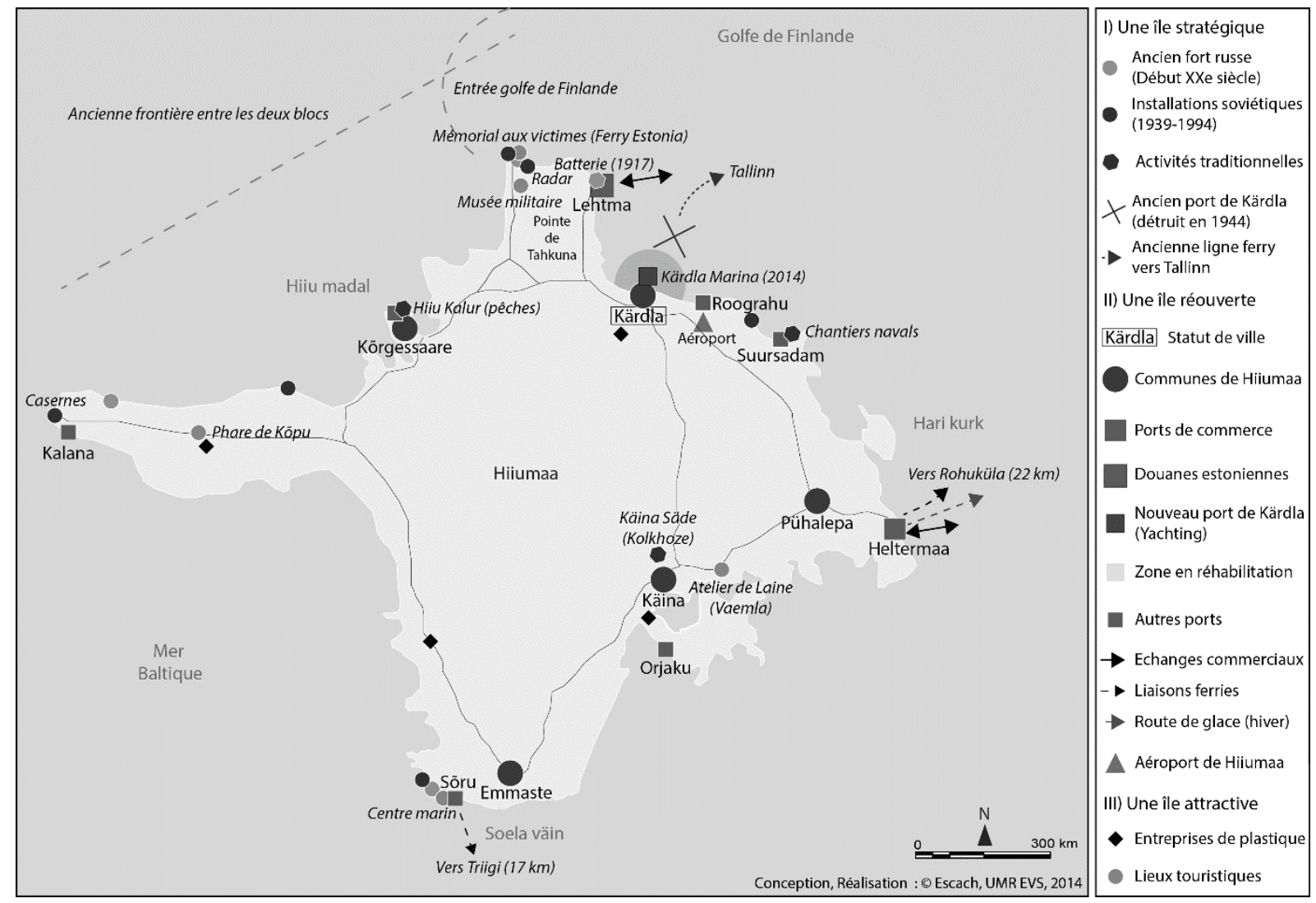

Dans un contexte plus urbain, le quartier de Karosta au nord de Liepāja, troisième ville de Lettonie, a accueilli une base navale soviétique jusque dans les années 1990. Les dernières troupes russes, soit 40000 soldats, n'ont quitté le port qu'en 1994 [Le Bourhis, 2008]. Cette situation a longtemps empêché Liepāja de devenir un port commercial d'importance, ce qui explique sa faible place dans la hiérarchie portuaire actuelle. Aujourd'hui, le quartier de Karosta et le canal qui le traverse présentent de graves problèmes de pollution, les Russes ayant coulé la plupart des navires avant leur départ.

\section{Une relative pacification avec la fin de la guerre froide}

À la fin de la guerre froide, les États baltes ont choisi la méthode de l'équidistance pour le tracé de leurs frontières maritimes respectives ainsi qu'avec la Suède et la Finlande ${ }^{5}$ [Ortolland, Pirat, 2010]. Des litiges ont en revanche opposé la Lettonie et la Lituanie au sujet de leur frontière commune. Fin 1995, le gouvernement letton a en effet signé un accord avec deux compagnies pétrolières étrangères pour exploiter des puits de pétrole offshore en mer Baltique au sein d'une zone disputée. De son côté, la Lituanie prévoyait la construction d'un terminal pétrolier à Būtingè [Chillaud, 2007] fortement critiqué par son voisin au nom d'arguments écologiques.

\footnotetext{
${ }^{5}$ Un accord d'avril 1997 a établi un tripoint équidistant des côtes de Lettonie, de Suède et d'Estonie. En 2001, la même opération a été répétée pour déterminer un tripoint équidistant des côtes d'Estonie, de Finlande et de Suède.
} 
Les négociations ont finalement abouti sous la pression de 1'Union européenne en mai $1999^{6}$. La fixation de la frontière maritime entre Estonie et Russie a quant à elle été retardée par un contentieux terrestre au niveau du lac Peïpous. Un accord a été engagé en février 2014 sur un échange de 128,5 hectares de terre et $11,4 \mathrm{~km}^{2}$ de surface aquatique dans le lac ainsi que sur une ligne de démarcation dans la baie de Narva et le golfe de Finlande. Dans les passages resserrés, des ententes ont pu être trouvées afin de maintenir une zone de mer internationale. L'Estonie et la Finlande ont décidé en 1994 de borner leurs mers territoriales dans le golfe de Finlande à 3 milles nautiques de la ligne médiane, ce qui revenait à laisser libre un corridor de 6 milles nautiques utilisé d'ailleurs par le gazoduc Nord Stream ainsi que par le trafic maritime en direction de la Russie. Une mesure similaire a été adoptée entre Bornholm et la Suède et dans les détroits danois.

Deux différends territoriaux persistent cependant sous forme de conflits larvés. Le Danemark et la Pologne n'ont toujours pas délimité leur frontière maritime entre l'île de Bornholm et la côte polonaise, ce qui a suscité des incertitudes pour le tracé du gazoduc Nord Stream. L'approche septentrionale des ports de Świnoujście et de Szczecin fait également l'objet d'une contestation entre Allemagne et Pologne, l'Allemagne considérant cette zone comme une partie de sa ZEE, la Pologne comme incluse dans ses eaux territoriales.

\section{La Baltique, un modèle de guerre hybride}

Si l'espace baltique est pacifié du point de vue des affrontements directs, les deux dernières décennies se sont caractérisées par une «guerre hybride » avec forte composante de soft power (désinformation à travers les médias, encouragement à la subversion politique, violation des espaces maritimes et aériens). Le dernier soldat russe n'a quitté le sol balte qu'en octobre 1999. Le souvenir de la présence russe est donc particulièrement présent pour les États baltes qui n'ont pas tardé à rejoindre l'OTAN en 2004. Ces adhésions ont été particulièrement mal perçues par le gouvernement russe qui multiplie les intimidations sans déclencher la clause de défense collective prévue par l'article 5 du traité de l'Atlantique Nord. La mer Baltique constitue donc une nouvelle ligne de démarcation entre les pays de l'UE membres ou non de l'OTAN à l'ouest et les pays satellites de la Russie qui souhaite étendre l'Union économique eurasiatique vers l'est.

Les conflits entre Russie et États baltiques membres de l'UE se sont notamment révélés à propos du passage du gazoduc Nord Stream dont le tracé forme géographiquement une sorte de nouveau Rideau de fer au centre de la Baltique ( $\mathrm{cf}$ figure $\mathrm{n}^{\circ} 1$ ). La construction du gazoduc a été décidée suite à un accord signé en 2005 entre Vladimir Poutine et Gerhard Schröder. Inauguré en 2011, sa mise en service a eu lieu en 2012, les deux pipes sous-marins parallèles reliant la région de Vyborg (Russie) à celle de Greifswald (Allemagne) sur 1198 kilomètres. Dès sa conception, le tracé du gazoduc achoppe [Cameron, 2007 ; Larsson, 2007]. Le premier sujet de discorde est politique. Il oppose la Russie aux traditionnels pays de transit (Pologne, Ukraine, Biélorussie, Slovaquie, République Tchèque) qui craignent une remise en cause de leur rôle et une extension de la guerre du gaz russo-ukrainienne à d'autres pays. Le tracé définitif évite la Pologne en raison de différends territoriaux non tranchés au niveau de Bornholm tout comme l'Estonie qui a refusé d'accueillir les recherches préalables sur ses eaux. La discorde politique s'est accompagnée d'inquiétudes quant à une instrumentalisation géostratégique voire militaire du gazoduc. Vladimir Poutine avait en effet annoncé en 2006 que le rôle de la Flotte russe était de protéger les intérêts économiques du pays en mer Baltique, par exemple la protection des gazoducs. Des experts et le ministre de la Défense suédois ont

\footnotetext{
${ }^{6}$ Accord du 9 juillet 1999 sur la délimitation des mers territoriales, des ZEE et des plateaux continentaux.
} 
exprimé leurs craintes sur de nouvelles intrusions de patrouilles ou sous-marins russes dans la ZEE suédoise ou l'utilisation du pipeline à des fins de renseignement ou d'espionnage au détriment des pays nordiques ou de l'OTAN grâce à des plateformes capteurs. Une troisième controverse a porté sur la question environnementale, non exempte d'égoïsme territorial sous la forme d'un syndrome NIMBY. Des ONG suédoises ainsi que l'Agence suédoise de protection de l'environnement ont fait part de leur inquiétude quant au passage du gazoduc à proximité d'une réserve marine près de Gotland. La branche finlandaise du WWF a appelé quant à elle à un tracé au sud de l'île d'Hogland arguant que le sol marin y était plus plat, ce qui permettrait un tracé plus droit du gazoduc et moins de risques de fuite. Le consortium du gazoduc a répondu que cette proposition était impossible compte tenu d'un projet de zone de conservation écologique. Enfin, la branche russe de Greenpeace a souligné la fragilité de l'est du golfe de Finlande, notamment la réserve Kurgalsky. L'argument écologique s'est doublé d'une crainte de risque technologique émise par les Suédois et Finlandais en raison des munitions enfouies dans le golfe de Finlande après la Seconde Guerre mondiale.

Après l'inauguration de Nord Stream, un autre élément récurrent de conflit a été la violation, supposée ou réelle, de l'intégrité territoriale notamment suédoise par l'armée russe. Régulièrement, des articles de presse évoquent une présence supposée de sous-marins russes, témoins oculaires à l'appui, et mettent en scène les recherches actives côté suédois sans que celles-ci n'aboutissent toujours à des faits avérés. En octobre 2014, une opération militaire a par exemple été lancée à la recherche d'un sous-marin russe de classe Lada endommagé autour de Kanholmsfjärden dans l'archipel de Stockholm. Cette «chasse aux sous-marins »a gagné d'autres pays comme la Finlande (avril 2015), la Lettonie (mai 2015, avril 2016) ou encore la Lituanie. En mai 2015, le gouvernement lituanien a ainsi accusé la Russie de rompre la Convention des Nations unies sur le droit de la mer en effectuant des rotations de navires dans la zone de construction de l'interconnexion électrique sous-marine NordBalt (Lituanie-Suède) au sein de la ZEE lituanienne. Les rumeurs de mouvements de bâtiments battant pavillon russe sont prises très au sérieux par les États baltes, d'autant plus depuis la crise ukrainienne qui a pu laisser craindre une intensification de la présence militaire russe en mer Baltique. Des incidents aériens ont également eu lieu comme en 2016 lorsque deux avions de combat russes Sukhoi Su24 ont réalisé entre quinze et vingt passes à très basse altitude au-dessus du navire de guerre américain USS Donald Cook au large de l'Estonie. Côté russe, une même rhétorique de provocation est employée à propos des exercices effectués par les navires de l'OTAN nommés Baltops. Les tensions se sont renforcés après les déclarations du ministre letton de la Défense se disant prêt en 2014 à accueillir des navires de l'OTAN à Liepāja au sein de la base navale.

Les tensions entre États riverains, OTAN et Russie se sont cristallisées autour de plusieurs hautlieux stratégiques : l'archipel de Stockholm, la baie de Gdańsk et la lagune de la Vistule, l'enclave de Kaliningrad et plus récemment l'île de Gotland. Les navires transitant le long des littoraux baltes et accusés de pénétrer dans les eaux baltes effectuent le plus souvent la liaison entre la base navale de Kronstadt (golfe de Finlande) située à proximité du Quartier général de la marine russe de Saint-Pétersbourg et la base de Baltiisk près du Quartier général de la Flotte de la Baltique à Kaliningrad. Cette route, le long du littoral balte, concentre d'ailleurs la plupart des accidents impliquant l'OTAN et la Russie comme le montre la figure $\mathrm{n}^{\circ} 3$. 


\section{Figure $n^{\circ} 3$ : Incidents impliquant l’OTAN et la Russie}

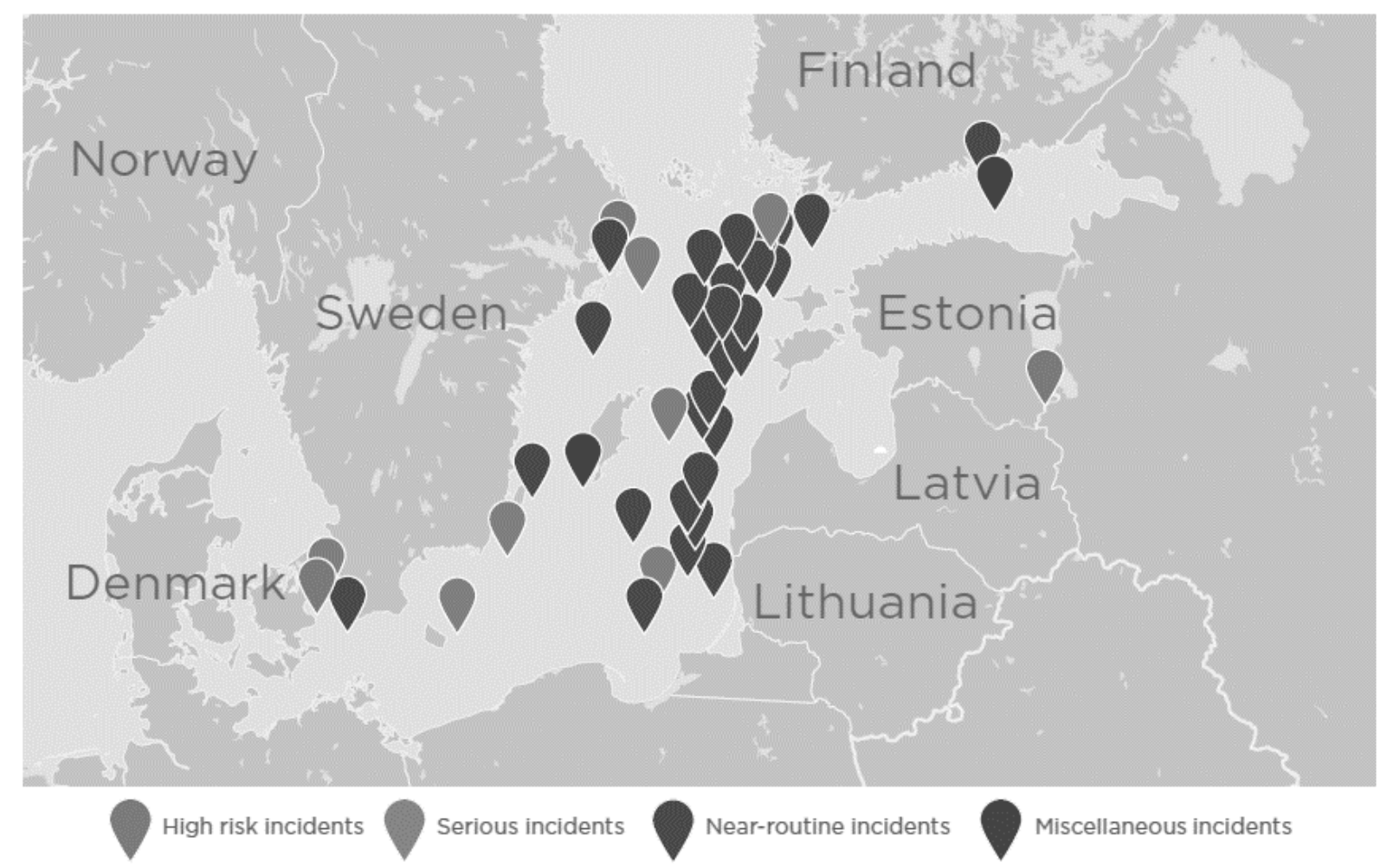

Source: European Leadership Network.

Source : Kramer, Nordenman, 2016

L'enclave de Kaliningrad constitue un point stratégique pour la Russie. La Flotte baltique comprend à Baltiisk deux sous-marins, deux destroyers, trois frégates, huit corvettes, un navire amphibie et des navires militaires côtiers. Le dispositif russe est constitué d'unités de défense côtières avec système d'alerte antimissile, station radar, et missiles Iskander positionnés à Chernyakhovsk, à $200 \mathrm{~km}$ de la base terrestre polonaise de Redzikowo. Cette dernière devrait abriter en 2018 une base du système de défense antimissile de l'OTAN. En 2014, la Russie a également déployé dans l'enclave des bombardiers tactiques Soukhoï Su-34 avec des capacités de lutte anti-navire (missiles Kh-35). De l'autre côté de la frontière, la baie de Poméranie accueille régulièrement des exercices Baltops, notamment les ports polonais de Gdynia ou Ustka $^{7}$. L'île de Gotland est également devenue plus récemment un lieu majeur de tensions en raison de sa situation stratégique (passage de Nord Stream, relais sur les axes maritimes majeurs). Le ministre de la Défense suédois a annoncé vouloir y poster 150 soldats pour faire face à une éventuelle action militaire russe dans la région sur le modèle de l'invasion de la Crimée. L'île a également accueilli plusieurs exercices de l'OTAN en 2015, soit un contingent de 3600 soldats et 115 avions. La perspective d'un gazoduc Nord Stream 2 a enfin relancé les débats dans la région autour du choix d'un futur tracé.

D'autres points d'achoppement sont apparus au moment de la crise ukrainienne affectant par exemple la commercialisation des ressources halieutiques baltes. Suite aux sanctions touchant la Russie, un embargo sur la plupart des produits alimentaires venant de l'Union européenne a été décrété par le Kremlin le 6 août 2014 pour une durée d'un an et étendu aux abats et farines

\footnotetext{
${ }^{7}$ L'OTAN a récemment reconnu l'importance stratégique des mers au sommet du Pays de Galles en septembre 2014 et la réalisation d'un cadre stratégique pour la mer Baltique est régulièrement mentionnée [Kramer, Nordenman, 2016].
} 
animales en octobre. Le 4 juin 2015, la Russie a imposé un nouvel embargo sur les poissons ou produits de la mer importés d'Estonie et de Lettonie, alors que près de la moitié de la production lettone se dirige vers le marché russe. Cette décision a fait suite à la découverte annoncée d'un taux excessif de Benzopyrène, une substance cancérigène, dans les sprats lettons par le Service Fédéral russe pour la Surveillance Vétérinaire et Phytosanitaire (Rosselkhoznadzor). Les sanctions russes n'ont cependant affecté que $0,5 \%$ du total des exportations lettones en 2014 selon l'OCDE.

\section{Des conflits sur la mer aux tensions pour la mer}

Les conflits géopolitiques interétatiques ne sont pas sans conséquences sur des conflits plus locaux générés par une appropriation croissante du territoire liquide [Escach, 2016]. La mer Baltique associe densité des usages et faible ressource spatiale, une combinaison propice aux tensions à toutes les échelles. Le trafic maritime la traversant représente par exemple $8 \% \mathrm{du}$ commerce maritime mondial en 2012, ce qui s'explique par un fort niveau d'internationalisation des économies baltiques et par la présence de clusters industriels dans la région et son hinterland. Celui-ci a doublé depuis 1997 alors que le trafic mondial ne connaissait qu'une croissance de $65 \%$ environ. S'ajoute au transit des porte-conteneurs à destination de SaintPétersbourg ou Gdańsk, de nombreuses lignes roulières locales ${ }^{8}$ (pour $44 \%$ d'entre elles), transnationales $(30 \%)$ ou à destination de la Norvège et de la Northern Range (26\%). Parallèlement, le tourisme de croisière s'est lui aussi développé rapidement (4,3 millions de passagers en 2012 contre seulement 1,1 millions en 2000) et représentait 10\% des parts de marché européennes en 2012. Cette superposition des lignes explique des phénomènes de saturation dans les passages resserrés à l'image du golfe de Finlande. Dans la baie d'Helsinki, les bacs vers Suomenlinna croisent les ferrys vers Tallinn ou Stockholm et les navires de croisière. Les activités traditionnelles, qui avaient généré une forte littoralisation sur la plupart des rives baltiques (tourisme balnéaire, pêche et aquaculture, transit portuaire, extraction de graviers), sont désormais débordées par de nouveaux usages à distance des côtes (énergies marines renouvelables, développement des croisières...). Comme le montre la figure $\mathrm{n}^{\circ} 1$, la plupart des activités se concentrent dans les mêmes zones : une polarisation nette au sud-ouest de la Baltique, au niveau des détroits danois et du littoral nord-allemand, où les conflits d'usage sont nombreux, et une concentration assez importante dans le golfe de Finlande plutôt caractérisé par des conflits géopolitiques à petite échelle.

Afin d'organiser le franchissement de la dernière frontière [Virilio, 2013], les activités humaines se déployant de plus en plus loin des terres, et parallèlement à des initiatives étatiques notamment en mer du Nord ${ }^{9}, 1^{\prime}$ UNESCO et l'UE ont successivement appelé à un aménagement de l'espace maritime. Une directive adoptée en 2014 a exhorté tous les États membres de l'UE à lancer un processus de planification dès septembre 2016 pour un aboutissement à l'horizon 2021. La planification maritime est à entendre ici comme un processus consistant à organiser géographiquement un espace maritime en rationalisant la localisation des activités humaines notamment par le zonage, chaque fraction délimitée se voyant attribuer des qualités spécifiques (types d'activités, réglementations, prescriptions). Les objectifs affichés par la Commission sont de plusieurs ordres: assurer localement une organisation plus rationnelle entre les différents usages de la mer, créer des synergies et réduire les conflits entre acteurs et entre États, appliquer à l'échelle européenne les objectifs de la stratégie Europe 2020 (encouragement de la

\footnotetext{
${ }^{8}$ Lignes au sein d'une même zone transfrontalière : Øresund, Baltique méridionale ou golfe de Finlande.

${ }^{9}$ En Europe, les pays du Nord comme la Belgique, le Royaume-Uni et l'Allemagne figurent parmi les pionniers en raison de la combinaison entre des espaces maritimes restreints et une forte convoitise des acteurs (ressources offshore, flux maritimes, pêche).
} 
recherche et des technologies numériques, essor d'une croissance bleue), renforcer enfin, à petite échelle, l'attractivité de l'espace maritime européen (susciter des investissements en accroissant leur prévisibilité, simplifier le processus de décision multiniveau, améliorer l'attractivité par une attention portée au paysage).

Si la planification de l'ensemble de la Baltique est encore embryonnaire, les exemples d'expériences menées à l'échelle locale existent. La planification maritime est inscrite dans la stratégie européenne pour la mer Baltique ratifiée en 2009 au sein de l'action horizontale consacrée à l'aménagement terrestre et maritime. Elle est encouragée par les organisations VASAB $^{10}$ et HELCOM qui ont conjointement défini en 2010 les principes d'une planification en Baltique. Plusieurs projets transnationaux ont vu le jour en Baltique pour entamer des études prospectives à l'image des projets INTERREG BaltSeaPlan (2009-2012) et PartiSEApate $(2012-2014)^{11}$. Ces démarches liminaires révèlent l'existence de multiples conflits d'usage et d'acteurs et la difficile conciliation entre des intérêts nationaux souvent divergents.

Une réflexion sur la planification de la ZEE lituanienne a été intégrée dans les projets BaltSeaPlan et PartiSEApate. La ZEE lituanienne fait partie des plus petites du monde et à fortiori de l'espace baltique avec une superficie de $7031 \mathrm{~km}^{2}$. Celle-ci est en grande partie organisée autour des mouvements au départ et à l'arrivée du port de Klaipéda qui concentre la majeure partie du trafic portuaire lituanien, les autres pôles côtiers étant le terminal pétrolier de Būtingè (d'où part un oléoduc) et le port de Šventoji (d'où partent deux câbles sous-marins ${ }^{12}$ ). Au sud de la ZEE, l'aménagement est contraint par des zones Natura 2000 de protection spéciale (ZPS) et de conservation (ZSC) s'inscrivant dans l'écosystème de la Lagune de Courlande classée à l'UNESCO. Deux zones militaires à accès restreint sont également situées dans la ZEE (EYD17 Juodkrantè, EYD18 Nida) auxquelles s'ajoutent des espaces identifiés comme dangereux en raison de la présence de mines. À l'extrême ouest de la zone, un site contenant des armes chimiques doit être préservé de toute activité de pêche notamment par les chalutiers.

De nouvelles activités doivent pourtant être développées au sein de la ZEE dans le futur. La stratégie nationale sur l'énergie 2008-2012 prévoit que la part des énergies renouvelables dans les énergies primaires au niveau national soit de $20 \%$ au moins d'ici à 2025. Deux parcs sont ainsi prévus (financés par JSC Renerga et Baltic Energy Groupe) pour une puissance de 800 MW. Parallèlement, un nouveau terminal flottant de stockage du LNG (gaz non liquéfié) nommé « Indépendance » (Independence) a débuté ses activités à Klaipėda le 3 décembre 2014. Il permet le stockage d'un gaz produit à Snøhvit en Norvège par la compagnie Statoil (compagnie étatique norvégienne). Ces choix actuels ou futurs, conformes au plan d'interconnexion des marchés énergétiques de la Baltique (PIMERB), ne manqueront pas de renforcer les tensions avec la Russie. La situation n'est pas très éloignée du pitch de la série Occupied de Jo Nesbø imaginant un conflit entre la Russie et la Norvège autour du contrôle de la production énergétique.

Le processus s'est révélé plus avancé dans la zone de la Baltique méridionale. Elle comprend sur $14100 \mathrm{~km}^{2}$ les eaux territoriales et les ZEE de plusieurs États (Allemagne, Pologne, Danemark, Suède), ce qui complique le processus d'expérimentation.

\footnotetext{
${ }^{10}$ VASAB est un forum de coopération associant les ministres responsables de l'aménagement du territoire de onze États (Norvège, Suède, Finlande, Russie, Estonie, Lettonie, Lituanie, Biélorussie, Pologne, Allemagne, Danemark) autour d'une démarche principalement prospective visant à définir des options possibles pour un développement territorial intégré de l'espace baltique.

${ }^{11} \mathrm{La}$ mer Baltique figure parmi les zones pilotes choisies par la Commission européenne avec la mer du Nord et la mer Adriatique.

${ }^{12}$ Le BCS East-West Interlink (en service depuis 1997) et le BCS East (en service depuis 1995).
} 
La difficulté d'une gouvernance commune est renforcée par des cultures de la planification et des systèmes politiques différents. La ZEE allemande et le littoral du MecklembourgPoméranie-Occidentale ont déjà fait l'objet de plans maritimes antérieurs qui doivent être intégrés, ce qui n'est pas le cas du Danemark et de la Suède. Le Land du MecklembourgPoméranie-Occidentale, très attractif pour les touristes (11\% du PIB), a déjà réalisé un plan régional pour la zone des 12 milles nautiques (eaux territoriales) prévoyant des règles particulièrement drastiques limitant l'implantation d'éoliennes offshore dans son secteur. Cela obligerait les autres États à accepter, dans le cadre d'une planification conjointe, plusieurs champs d'éoliennes en dehors des eaux territoriales allemandes. La situation rappelle à l'échelle étatique les dérives du phénomène NIMBY (Not In My BackYard).

Les conflits d'acteurs sont d'autant plus nombreux que la zone de la Baltique méridionale est écologiquement l'une des dix plus importantes aires d'habitat, d'hibernation, de mue et de migration des oiseaux de l'espace baltique. Plusieurs espèces sont représentées : canards de mer, mouette pygmée, macreuse noire, macreuse brune, harelde kakawi, plongeon catmarin, grèbe esclavon... Les zones de l'Oderbank et de l'Adlergrund sont les plus recherchées en raison d'eaux peu profondes (moins de 15-20 m) et de la présence de bancs de moules. La région est située à l'intersection de plusieurs itinéraires de migration (route est/ouest utilisée par les canards et les oies dans la partie sud du projet, déplacement des grues depuis Rügen jusqu'aux côtes de Scanie, route nord/sud pour les oiseaux terrestres). La présence d'espères protégées a des conséquences notables sur l'aménagement de la zone. Tout d'abord, les oiseaux se déplacent en permanence ce qui va à l'encontre de l'organisation par fractions d'espace. De plus, les blocs de glace qui dérivent en hiver sont autant de vecteurs modifiant la géographie de l'habitat des oiseaux. Enfin, selon les ornithologues, $30 \%$ environ des oiseaux volent en dessous de $200 \mathrm{~m}$ de hauteur, sont donc difficilement repérables par des radars et peuvent subir les conséquences d'installations de fermes éoliennes offshore. Un conflit d'usage entre production d'énergie et protection des espèces menacées risque ainsi d'émerger dans la zone car celle-ci est particulièrement propice à une exploitation de la force éolienne. La vitesse du vent à $100 \mathrm{~m}$ au-dessus du niveau de la mer peut même atteindre régulièrement le niveau 9 sur l'échelle de Beaufort (Fort coup de vent, 75 à 88 $\mathrm{km} / \mathrm{h}$ ) au niveau du bassin de Bornholm, à proximité de la zone contestée entre Pologne et Danemark. Outre les fermes éoliennes, la Baltique méridionale est une zone majeure pour l'extraction du sable et des graviers. Des licences d'exploitation ont notamment été accordées au niveau de la région de l'Adlergrund dans les eaux territoriales allemandes.

À l'issue de la concertation avec des acteurs locaux, les porteurs de projet ont conclu à quatre conflits d'usage possibles : entre la navigation et l'exploitation d'éoliennes, entre la protection environnementale et trois activités concurrentes que sont l'exploitation éolienne, l'extraction de sables et de graviers et les activités liées à la pêche ou la plaisance. Afin de contourner la difficulté, les coordinateurs ont proposé d'associer deux systèmes : le zoning d'une part et l'approche sectorielle d'autre part. Le tout est modélisé grâce à un logiciel d'aide à la décision nommé Marxan. Les activités ne répondant pas à une logique de site et impliquant des territoires aux limites fluctuantes comme la pêche semblent être les perdantes d'un tel quadrillage malgré leur importance décisive pour le développement des populations côtières.

Les zones définies in fine sont de quatre types : les zones prioritaires (aucune activité portant atteinte à l'usage défini comme prioritaire ne sera permise), les zones réservées (au sein desquelles certains usages sont avantagés), les zones ou domaines appropriés (un usage est déterminé pour ces zones et interdit ailleurs) et les zones à libre usage (toutes les activités sont tolérées). Les zones les plus contraignantes sont en réalité rarement instituées, y compris lorsqu'il s'agit de protéger l'environnement. La pêche, le tourisme, l'exploitation du sable et graviers et les réglementations Natura 2000 s'exercent au sein de zones réservées : pas de 
priorité absolue, mais simplement une "attention particulière » dans l'arbitrage avec d'autres usages. Seuls les parcs nationaux sont classés comme zones prioritaires.

\section{Conclusion}

Loin de constituer l'apogée d'un rapprochement attendu, la gestion commune du milieu marin, bien que fondamentale, a plutôt contribué à l'émergence d'un consensus mou, alors que des questions sécuritaires et géopolitiques majeures restaient non résolues. Des normes plus ou moins respectées censées prévenir les risques, lutter contre la pollution ou réguler le trafic maritime ont relégué au second plan des débats jugés trop polémogènes. En ce sens, les États riverains et les autorités européennes ont échoué, malgré une configuration favorable, à inventer des interactions innovantes et pacifiées entre l'UE et la Russie, par exemple à propos de Kaliningrad. Le Conseil des États de la mer Baltique, créé en 1992, associant la Russie aux États baltes et aux autres pays riverains, a rapidement écarté les questions de sécurités dures au profit d'une coopération sur des sujets moins polémiques comme l'environnement, la culture ou l'économie [Marin, 2009]. La stratégie européenne en mer baltique, censée renforcer la Dimension septentrionale en approfondissant la gouvernance transfrontalière aux marges de l'Europe, a quant à elle rapidement relayé la Russie au rang de "partenaire associé ». Les conflits d'usage ne pourront être efficacement traités sans des coopérations actives avec la Russie et la Biélorussie qui comptent parmi les pays les plus pollueurs. La Baltique n'aurait-telle donc rien d'autre à partager que les conséquences de la «tragédie des biens communs ${ }^{13}$ ?

\section{Bibliographie}

BRUNET R. (1995), « Modèles de Méditerranées », L'Espace Géographique, 24 (3), 200-202.

CAMERON F. (2007), « Le projet de gazoduc Nord Stream et ses implications stratégiques », Note de la Direction générale des politiques internes, Département thématique $\mathrm{C}$ : droit des citoyens et affaires constitutionnelles, Bruxelles, Parlement européen, 17 pages.

CHILLAUD M. (2007), La démarche stratégique des États baltes dans l'architecture européenne de sécurité et de défense. Une politique fondée sur une dialectique identitaire et militaire, Thèse soutenue à l'université Montesquieu de Bordeaux le 30 mars 2007, 613 p.

CHRISTIANSEN T. (1997), «A European Meso-Region? European Perspectives on the Baltic Sea Region » in: Neo-nationalism or regionality, the restructuring of political space around the Baltic Rim, Stockholm, NordREFO, 254-292.

COMMISSION EUROPEENNE, (1994), Europe 2000 +, Coopération pour l'aménagement du territoire européen, Luxembourg, Office des publications officielles des communautés européennes, $244 \mathrm{p}$.

COMMISSION EUROPEENNE, (1999), Schéma de Développement de l'Espace Communautaire, Luxembourg, Office des publications officielles des communautés européennes, $94 \mathrm{p}$.

DOLLFUS O. (1995), «Méditerranées, essai d'analyse géographique », L’Espace géographique, 24 (3), 194-199.

\footnotetext{
${ }^{13}$ Expression tirée d'un article controversé de l'écologue américain Garrett James Hardin publié dans la revue Science en 1968.
} 
ESCACH N., (dir.), (2014), Géographie des mers et des océans, Paris, Dunod, 448 p.

ESCACH N. (2016), « De la mer en partage au partage de la mer », Monde diplomatique, juillet 2016.

HELCOM, (1974), Convention of the protection of the marine environment of the Baltic Sea Area, Helsinki, disponible en ligne: http://www.helcom.fi (consulté le 20/08/2013).

JOENNIEMI P. (2009), "The EU Strategy for the Baltic Sea Region, a catalyst for what? », DIIS Brief, Danish institute for international studies, 8 p.

KNUDSEN O. (dir.), (1999), Stability and Security in the Baltic Sea Region, Londres/Portland, Frank Cass, 1999, 287 p.

KRAMER F-D., NORDENMAN M. (2016), «A Maritime Framework for the Baltic Sea Region », Issue Brief, Brent Scowcroft Centeron International Security, Atlantic Council, 11 p.

LARSSON R-L. (2007), « Nord Stream, Sweden and Baltic Sea Security », Defense Analysis, FOI, Swedish Defense Research Agency, 110 p.

LE BOURHIS E. (2008), « Karosta, du militaire au culturel », Regard sur l'Est, disponible en ligne.

MARIN A. (2009), «Argument Baltique : faux prétexte et modèle juste », Outre-Terre, 3 (23), $347-362$.

ORTOLLAND D., PIRAT J-P. (2010), Atlas géopolitique des espaces maritimes, deuxième édition, Éditions Technip, Paris, 333 p.

OSTERGREN R., LE BOSSÉ M. (2005), «Cross-border Regionalism and the "Macro" Scale: the Baltic Sea Region », in: Regionalism in the Age of Globalism. vol. 2: Forms of Regionalism, Madison, University of Wisconsin Press, 49-76.

RYDÉN L. (2002), «The Baltic Sea Region and the relevance of regional approaches », in: The Baltic Sea Region, Cultures, Politics, Societies, Uppsala, The Baltic University Press, 7-29.

VIRILIO P. (2013), Le littoral, la dernière frontière, entretien avec Jean-Louis Violeau, Paris, Sens \& Tonka, 42 p. 